\title{
Promises of Conic Relaxation for Contingency-Constrained Optimal Power Flow Problem
}

\author{
Ramtin Madani, Morteza Ashraphijuo and Javad Lavaei
}

\begin{abstract}
This paper is concerned with the securityconstrained optimal power flow (SCOPF) problem, where each contingency corresponds to the outage of an arbitrary number of lines and generators. The problem is studied by means of a convex relaxation, named semidefinite program (SDP). The existence of a rank-1 SDP solution guarantees the recovery of a global solution of SCOPF. We prove that the rank of the SDP solution is upper bounded by the treewidth of the power network plus one, which is perceived to be small in practice. We then propose a decomposition method to reduce the computational complexity of the relaxation. In the case where the relaxation is not exact, we develop a graph-theoretic convex program to identify the problematic lines of the network and incorporate the loss over those lines into the objective as a penalization (regularization) term, leading to a penalized SDP problem. We perform several simulations on large-scale benchmark systems and verify that the global minima are at most $1 \%$ away from the feasible solutions obtained from the proposed penalized relaxation.
\end{abstract}

\section{INTRODUCTION}

The classical optimal power flow (OPF) problem aims to find a steady-state operating point of a power system that minimizes a desirable cost function, e.g. power loss or generation cost, and satisfies network and physical constraints on loads, powers, voltages and line flows [1]. The OPF problem is not only non-convex but also NP-hard, due to its possible reduction to the $(0,1)$-quadratic optimization. Started by the work [2] in 1962, many of the existing optimization techniques have been studied for the OPF problem, leading to algorithms based on linear programming, Newton Raphson, quadratic programming, nonlinear programming, Lagrange relaxation, interior point method, artificial intelligence, artificial neural network, fuzzy logic, genetic algorithm, evolutionary programming and particle swarm optimization [3]. Due to the non-convexity of OPF, these algorithms are not robust, lack performance guarantees, and may not find a global optimum.

Followed by the idea proposed in [4] and by exploiting the physical properties of transmission lines, it has been argued in the series of work [5], [6], [7], [8], [9], [10], [11], [12] that the classical OPF problem corresponding to a practical power system may be convexified and solved efficiently through a semidefinite programming (SDP) relaxation. In particular, the paper [13] shows that the SDP relaxation is exact in two cases under certain technical assumptions: (i) for acyclic networks, (ii) for cyclic networks after relaxing the angle constraints. However, the SDP relaxation is not always exact for a general mesh network [14], [15], [16], [17]. This issue has been discussed extensively in the literature and several test cases

The authors are with the Electrical Engineering Department, Columbia University (email: madani@ee.columbia.edu, ashraphijuo@ee.columbia.edu and lavaei@ee.columbia.edu). This work was supported by NSF CAREER Award 1351279, NSF EECS Award 1406865, and ONR YIP Award. have been contrived that witness the failure of SDP relaxation in obtaining a global optimal solution of the OPF problem [15], [18]. To ameliorate the issue, we have recently shown in [19] that: (i) the exactness of the SDP relaxation depends on the formulation of the line capacity constraints, and (ii) the penalization of total reactive loss may enable the recovery of a near-global solution (i.e., a solution that is measurably close to a global minimum) for modest-sized systems (as verified in over 7000 simulations).

The major drawback of representing the optimal power flow problem as a semidefinite program is the requirement of defining a square matrix variable, which makes the number of scaler variables of the problem quadratic with respect to the number of network buses. This may yield a very high-dimensional SDP problem for a real-world network. To address this issue, the papers [20], [21], [22] have leveraged the sparsity of power networks in order to break down the large-scale semidefinite constraint into small-sized constraints. Similarly, the papers [23], [24] and [25] have exploited the general technique proposed in [26], [27] to reduce the complexity of the SDP relaxation of the OPF problem. The simulations performed in those papers suggest that the SDP relaxation would fail to work properly for large-scale systems [22].

Although OPF is a fundamental problem studied extensively in the literature for power systems, a real-world power flow optimization is based on a set of coupled OPFs with a variety of constraints and variables. The latter problem is named security-constrained OPF (SCOPF) [28], [29]. The SCOPF problem is important in practice, since independent system operators tend to design an operating point that satisfies the demand and network constraints not only under normal operation but also under pre-specified contingencies such as line outages. Depending on the network characteristics, one may adopt preventive or corrective approaches for the SCOPF problem. In the preventive formulation of SCOPF, the under-design state of each generator (e.g., production level) is considered identical for the pre- and post-contingency scenarios. This reflects the fact that mechanical facilities may not be able to respond to the changes in the network fast. In the corrective approach, limited changes in certain control parameters are permitted after the network experiences a fault. SCOPF is more challenging than the conventional OPF problem for two reasons. First, the size of the optimization could be prohibitive, depending on the number of contingencies. Second, SCOPF is obtained by coupling a group of non-convex OPF problems associated with different contingencies and therefore its non-convexity would be much higher than an individual OPF problem. The purpose of this work is to propose an efficient computational method that can be applied to not only OPF but also SCOPF.

In this paper, we study the SCOPF problem-as a general 
version of OPF - through a convex relaxation. First, we propose an SDP relaxation for this problem. The existence of a rank-1 SDP solution guarantees the recovery of a global solution of SCOPF. We prove that the relaxation has a matrix solution whose rank is at most the treewidth of the precontingency network plus one. The treewidth of real-world networks is perceived to be small due to their (almost) planarity and sparsity [30]. For example, the treewidth of the graph corresponding to a peak hour setup of a Polish system with over 3000 buses is less than 25 . Second, we reduce the computational complexity of the SDP problem using a tree decomposition method to arrive at a decomposed SDP relaxation with a set of small-sized SDP matrices as opposed to a full-scale SDP matrix. We show that the fullscale SDP relaxation has a solution whose rank is upper bounded by the ranks of the small-sized matrices of the decomposed SDP relaxation. By working on the ranks of these small matrices, we propose a technique to identify the problematic lines of the network for each contingency that may contribute to the inexactness of the SDP relaxation for SCOPF. This diagnosis method may enable us to develop a heuristic method, named penalized SDP relaxation, to find a near-global solution of the problem by penalizing the loss over the problematic lines for each contingency. Note that a certain line may be problematic with respect to one contingency and not problematic with respect to another contingency. It is suggested to define the penalty term as a summation of all loss functions for problematic lines under each contingency. Note that a uniform penalty — consisting of losses over all lines for all contingencies-also work for all test systems studied in this paper, but the resulting SDP solution would have a lower global optimality guarantee compared to the case where the loss over only problematic lines is penalized. We test our method on several benchmark systems with as high as 3000 buses and find a solution with a global optimality guarantee of at least $99 \%$ for each case.

Notations: $\mathbb{R}, \mathbb{C}$, and $\mathbb{H}^{n}$ denote the sets of real numbers, complex numbers, and $n \times n$ Hermitian matrices, respectively. The $m$ by $n$ rectangular identity matrix whose $(i, j)$ entry is equal to the Kronecker delta $\delta_{i j}$ is denoted by $\mathbf{I}_{m \times n}$. The notations $\operatorname{Re}\{\mathbf{W}\}, \operatorname{Im}\{\mathbf{W}\}$, and $\operatorname{rank}\{\mathbf{W}\}$ denote the real part, imaginary part, and rank of a scalar/matrix $\mathbf{W}$, respectively. The notation $\mathbf{W} \succeq 0$ means that $\mathbf{W}$ is Hermitian and positive semidefinite. The notation $\measuredangle x$ denotes the angle of a complex number $x$. The notation "i" is reserved for the imaginary unit. The superscripts $(\cdot)^{*}$ and $(\cdot)^{\mathrm{T}}$ represent the conjugate transpose and transpose operators, respectively. Given a matrix $\mathbf{W}$, its $(l, m)$ entry is denoted as $W_{l m}$. The subscript $(\cdot)_{\text {opt }}$ is used to show the optimal value of an optimization parameter. Given a matrix M, its Moore Penrose pseudoinverse is denoted as $\mathbf{M}^{+}$. Given a simple graph $\mathcal{H}$, its vertex and edge sets are denoted by $\mathcal{V}_{\mathcal{H}}$ and $\mathcal{E}_{\mathcal{H}}$, respectively. Given two sets $\mathcal{S}_{1}$ and $\mathcal{S}_{2}$, the notation $\mathcal{S}_{1} \backslash \mathcal{S}_{2}$ denotes the set of all elements of $\mathcal{S}_{1}$ that do not exist in $\mathcal{S}_{2}$. Given a scalar $m$ and a real-valued set $\mathcal{S}$, define $\mathcal{S}+m$ as a set obtained by adding $m$ to every element of $\mathcal{S}$. Given a Hermitian matrix $\mathbf{M}$ and two sets of natural numbers $\mathcal{S}_{1}$ and $\mathcal{S}_{2}$, define $\mathbf{M}\left\{\mathcal{S}_{\mathbf{1}}, \mathcal{S}_{\mathbf{2}}\right\}$
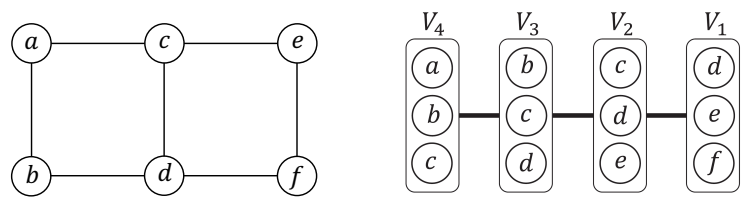

Fig. 1: A minimal tree decomposition for a ladder

as a submatrix of $\mathbf{M}$ obtained through two operations: (i) removing all rows of $\mathrm{M}$ whose indices do not belong to $\mathcal{S}_{1}$, and (ii) removing all columns of $\mathbf{M}$ whose indices do not belong to $\mathcal{S}_{2}$. For instance, $\mathbf{M}\{\{\mathbf{1}, \mathbf{2}\},\{\mathbf{2}, \mathbf{3}\}\}$ is a $2 \times 2$ matrix with the entries $M_{12}, M_{13}, M_{22}, M_{23}$.

\section{Problem fORmulation}

Consider a power network with the set of buses $\mathcal{N}:=$ $\{1,2, \ldots, n\}$ and the set of flow lines $\mathcal{L} \subseteq \mathcal{N} \times \mathcal{N}$. With no loss of generality, each line $(l, m) \in \mathcal{L}$ of the network is modeled as a series admittance $y_{l m}$. Suppose that a known constantpower load with the complex value $S_{D_{k}}=P_{D_{k}}+Q_{D_{k}} \mathrm{i}$ is connected to bus $k \in \mathcal{N}$, where $P_{D_{k}}, Q_{D_{k}} \in \mathbb{R}$. Given a nonnegative integer $c$, consider a set of $c$ contingencies, where each contingency corresponds to an arbitrary number of pre-specified line/generator outages. In this work, we model a line outage by removing the line from the base case and model a generator outage by enforcing its output to be zero. Define $\mathcal{C}:=\{0,1, \ldots, c\}$ as the set of all pre- and postcontingencies, where the base case is treated as contingency 0 . Define $\mathcal{L}^{(0)}=\mathcal{L}$, and $\mathcal{L}^{(r)}$ as the set of lines of the network under contingency $r \in\{1,2, \ldots, c\}$.

Consider a contingency scenario $r \in \mathcal{C}$. Assume that a generator is connected to each bus $k \in \mathcal{N}$, whose unknown complex output is denoted as $S_{G_{k}}^{(r)}=P_{G_{k}}^{(r)}+Q_{G_{k}}^{(r)}$ i. Let $f_{k}^{(r)}(\cdot)$ be a convex function representing the generation cost for generator $k$ in the contingency case $r \in \mathcal{C}$. The unknown complex voltage at bus $k \in \mathcal{N}$ is denoted as $V_{k}^{(r)}$. Furthermore, define $S_{l m}^{(r)}=P_{l m}^{(r)}+Q_{l m}^{(r)} \mathrm{i}$ as the unknown complex power transferred from bus $l \in \mathcal{N}$ to the rest of the network through the line $(l, m) \in \mathcal{L}^{(r)}$. Define:

$$
\begin{array}{rlrl}
\mathbf{P}_{G}^{(r)} & \triangleq\left[P_{G_{1}}^{(r)}, \ldots, P_{G_{n}}^{(r)}\right]^{\mathrm{T}}, & \mathbf{Q}_{G}^{(r)} \triangleq\left[Q_{G_{1}}^{(r)}, \ldots, Q_{G_{n}}^{(r)}\right]^{\mathrm{T}}, \\
\mathbf{P}_{D} \triangleq\left[P_{D_{1}}, \ldots, P_{D_{n}}\right]^{\mathrm{T}}, & \mathbf{Q}_{D} \triangleq\left[Q_{D_{1}}, \ldots, Q_{D_{n}}\right]^{\mathrm{T}}, \\
\mathbf{V}^{(r)} & \triangleq\left[V_{1}^{(r)}, \ldots, V_{n}^{(r)}\right]^{\mathrm{T}} . &
\end{array}
$$

Given the known vectors $\mathbf{P}_{D}$ and $\mathbf{Q}_{D}$, SCOPF minimizes the generation cost over the unknown parameters $\mathbf{V}^{(r)}, \mathbf{P}_{G}^{(r)}$ and $\mathbf{Q}_{G}^{(r)}$ for $r=0,1, \ldots, c$ subject to the power balance equations at all buses and certain network constraints. SCOPF is formalized below.

\section{SCOPF problem: Minimize}

$$
\sum_{r \in \mathcal{C}} \sum_{k \in \mathcal{N}} f_{k}^{(r)}\left(P_{G_{k}}^{(r)}\right)
$$

over the variables $\mathbf{P}_{G}^{(0)}, \mathbf{P}_{G}^{(1)}, \ldots, \mathbf{P}_{G}^{(c)} \in \mathbb{R}^{n}, \mathbf{Q}_{G}^{(0)}, \mathbf{Q}_{G}^{(1)}$, $\ldots, \mathbf{Q}_{G}^{(c)} \in \mathbb{R}^{n}$ and $\mathbf{V}^{(0)}, \mathbf{V}^{(1)}, \ldots, \mathbf{V}^{(c)} \in \mathbb{C}^{n}$, subject to 

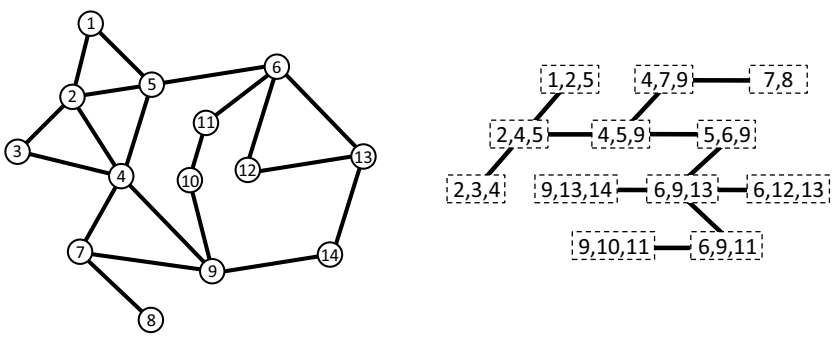

Fig. 2: The IEEE 14-bus test case and its minimal tree decomposition

$$
\begin{aligned}
& P_{G_{k}}^{(r)}-P_{D_{k}}=\sum_{l \in \mathcal{N}_{k}^{(r)}} \operatorname{Re}\left\{V_{k}^{(r)}\left(V_{k}^{(r)}-V_{l}^{(r)}\right)^{*} y_{k l}^{*}\right\} \\
& Q_{G_{k}}^{(r)}-Q_{D_{k}}=\sum_{l \in \mathcal{N}_{k}^{(r)}} \operatorname{Im}\left\{V_{k}^{(r)}\left(V_{k}^{(r)}-V_{l}^{(r)}\right)^{*} y_{k l}^{*}\right\} \\
& P_{k ; \min }^{(r)} \leq P_{G_{k}}^{(r)} \leq P_{k ; \max }^{(r)} \\
& Q_{k ; \min }^{(r)} \leq Q_{G_{k}}^{(r)} \leq Q_{k ; \max }^{(r)} \\
& V_{k ; \min }^{(r)} \leq\left|V_{k}^{(r)}\right| \leq V_{k ; \max }^{(r)} \\
& \left|\measuredangle V_{l}^{(r)}-\measuredangle V_{m}^{(r)}\right| \leq \theta_{l m ; \max }^{(r)} \\
& \left(P_{l m}^{(r)}\right)^{2}+\left(Q_{l m}^{(r)}\right)^{2} \leq\left(S_{l m ; \max }^{(r)}\right)^{2} \\
& \left|P_{G_{k}}^{(r)}-P_{G_{k}}^{(0)}\right| \leq \Delta P_{k ; \max }^{(r)} \\
& \left|Q_{G_{k}}^{(r)}-Q_{G_{k}}^{(0)}\right| \leq \Delta Q_{k ; \max }^{(r)} \\
& \left.|| V_{k}^{(r)}\right|^{2}-\left|V_{k}^{(0)}\right|^{2} \mid \leq\left(\Delta V_{k ; \max }^{(r)}\right)^{2}
\end{aligned}
$$

for every $k \in \mathcal{N}, r \in \mathcal{C}$ and $(l, m) \in \mathcal{L}^{(r)}$, where

- $\mathcal{N}_{k}^{(r)}$ denotes the set of all neighboring nodes of bus $k \in$ $\mathcal{N}$ for contingency $r$.

- (2a) and (2b) are the power balance equations accounting for the conservation of energy at bus $k$.

- (2c), (2d) and (2e) restrict the active power, reactive power and voltage magnitude at bus $k$ for contingency $r$, given the limits $P_{k ; \text { min }}^{(r)}, P_{k ; \max }^{(r)}, Q_{k ; \min }^{(r)}, Q_{k ; \max }^{(r)}, V_{k ; \min }^{(r)}$ and $V_{k ; \max }^{(r)}$.

- Each line $(l, m) \in \mathcal{L}^{(r)}$ of the network is subject to two capacity constraints. The constraint (2f) restricts the voltage phase difference of buses $l$ and $m$ with the limit $\theta_{l m ; \max }^{(r)} \in\left[0,90^{\circ}\right]$. Moreover, $(2 \mathrm{~g})$ restricts the apparent power flow $\left|S_{l m}^{(r)}\right|$ with the line capacity $S_{l m ; \max }^{(r)}$.

- Given the limits $\Delta P_{k ; \max }^{(r)}, \Delta Q_{k ; \max }^{(r)}$ and $\Delta V_{k ; \max }^{(r)}$, the constraints (2h), (2i) and (2j) ensure that the potentially controllable parameters $P_{G_{k}}^{(r)}, Q_{G_{k}}^{(r)}$ and $\left|V_{k}^{(r)}\right|$ vary within permissible ranges after a contingency occurs.

Note that a generator outage can be modeled as

$$
P_{k ; \min }^{(r)}=P_{k ; \max }^{(r)}=Q_{k ; \min }^{(r)}=Q_{k ; \max }^{(r)}=0 .
$$

\section{A. Convex relaxation for $S C O P F$}

The SCOPF problem includes quadratic constraints such as (2a) and (2b). Nevertheless, all constraints of (2) can be expressed linearly in terms of the entries of the matrix variable $\mathbf{W}$ defined as $\mathbf{W} \triangleq \mathbf{V V}^{*}$, where $\mathbf{V}$ denotes a column vector obtained by stacking the voltage vectors $\mathbf{V}^{(0)}, \mathbf{V}^{(1)}, \ldots, \mathbf{V}^{(c)}$. On the other hand, the variable $\mathbf{V}$ can be dropped from the optimization problem by equivalently replacing the consistency constraint $\mathbf{W}=\mathbf{V V}^{*}$ with two new constraints: (i) $\mathbf{W} \succeq 0$, and (ii) $\operatorname{rank}\{\mathbf{W}\}=1$. Observe that Constraint (ii) is the only non-convex constraint of the reformulated SCOPF problem. Motivated by this fact, the SDP relaxation of SCOPF is defined as the optimization problem reformulated in terms of $\mathbf{W}$ under the additional constraint $\mathbf{W} \succeq 0$ without incorporating the rank constraint $\operatorname{rank}\{\mathbf{W}\}=1$. To formalize this relaxation, let $\mathbf{W}^{(r)} \in \mathbb{H}^{n}$ denote the submatrix of $\mathbf{W}$ in the intersection of rows $r n+1, \ldots, r n+n$ with columns $r n+1, \ldots, r n+n$.

\section{Relaxed SCOPF: Minimize}

$$
\sum_{r \in \mathcal{C}} \sum_{k \in \mathcal{N}} f_{k}^{(r)}\left(P_{G_{k}}^{(r)}\right)
$$

over the parameters $\mathbf{P}_{G}^{(0)}, \mathbf{P}_{G}^{(1)}, \ldots, \mathbf{P}_{G}^{(c)} \in \mathbb{R}^{n}, \mathbf{Q}_{G}^{(0)}, \mathbf{Q}_{G}^{(1)}$, $\ldots, \mathbf{Q}_{G}^{(c)} \in \mathbb{R}^{n}$ and $\mathbf{W} \in \mathbb{H}^{n(c+1)}$, subject to

$$
\begin{aligned}
& P_{G_{k}}^{(r)}-P_{D_{k}}=\sum_{l \in \mathcal{N}_{k}^{(r)}} \operatorname{Re}\left\{\left(W_{k k}^{(r)}-W_{k l}^{(r)}\right) y_{k l}^{*}\right\} \\
& Q_{G_{k}}^{(r)}-Q_{D_{k}}=\sum_{l \in \mathcal{N}_{k}^{(r)}} \operatorname{Im}\left\{\left(W_{k k}^{(r)}-W_{k l}^{(r)}\right) y_{k l}^{*}\right\} \\
& P_{k ; \min }^{(r)} \leq P_{G_{k}}^{(r)} \leq P_{k ; \max }^{(r)} \\
& Q_{k ; \min }^{(r)} \leq Q_{G_{k}}^{(r)} \leq Q_{k ; \max }^{(r)} \\
& \left(V_{k ; \min }^{(r)}\right)^{2} \leq W_{k k}^{(r)} \leq\left(V_{k ; \max }^{(r)}\right)^{2} \\
& \operatorname{Im}\left\{W_{l m}^{(r)}\right\} \leq \operatorname{Re}\left\{W_{l m}^{(r)}\right\} \tan \left(\theta_{l m ; \max }^{(r)}\right) \\
& \left(P_{l m}^{(r)}\right)^{2}+\left(Q_{l m}^{(r)}\right)^{2} \leq\left(S_{l m ; \max }^{(r)}\right)^{2} \\
& \left|P_{G_{k}}^{(r)}-P_{G_{k}}^{(0)}\right| \leq \Delta P_{k ; \max }^{(r)} \\
& \left|Q_{G_{k}}^{(r)}-Q_{G_{k}}^{(0)}\right| \leq \Delta Q_{k ; \max }^{(r)} \\
& \left|W_{k k}^{(r)}-W_{k k}^{(0)}\right| \leq\left(\Delta V_{k ; \max }^{(r)}\right)^{2} \\
& \mathbf{W} \succeq 0
\end{aligned}
$$

for every $k \in \mathcal{N}, r \in \mathcal{C}$ and $(l, m) \in \mathcal{L}^{(r)}$.

The relaxed SCOPF is alternatively referred to as SDP relaxation henceforth. Let $f_{\text {opt }}$ and $f_{r-\text { opt }}$ denote the optimal objective values of the SCOPF and relaxed SCOPF. As shown in [6] and [31], the relaxed SCOPF is equivalent to the dual of the dual of the SCOPF problem and therefore it provides a lower bound $f_{r-\text { opt }}$ on the globally minimum solution $f_{\text {opt }}$ of the original problem (2). Hence, $f_{\mathrm{opt}}-f_{r-\mathrm{opt}}$ represents the duality gap for the non-convex SCOPF problem, which is not necessarily zero or even small [14], [15], [18]. Zero duality gap is a favorable property because of two reasons: (i) it guarantees the existence of a rank-1 solution $\mathbf{W}_{\text {opt }}$, (ii) it attains the optimal objective value of the SCOPF problem. If $\mathbf{W}_{\mathrm{opt}}$ is obtained numerically, an optimal vector of voltage phasors $\mathbf{V}_{\text {opt }}$ can then be constructed through the 
decomposition $\mathbf{W}_{\mathrm{opt}}=\mathbf{V}_{\mathrm{opt}} \mathbf{V}_{\mathrm{opt}}^{*}$. It has been shown in [31] that whenever the duality gap of the classical OPF problem is zero for a specific power network, the SCOPF problem also possesses zero duality gap, leading to the presence of a rank-1 solution for the relaxed SCOPF. As investigated in [32] and [13], the duality gap for the OPF problem (without any contingency scenarios) is highly correlated with the topology of the network. In addition, the gap heavily depends on the mathematical formulation of the line capacity constraints [19]. Since a power network has a sparse graph in general, the relaxed SCOPF problem may have infinitely many solutions. As an extreme case, the duality gap could be zero and yet there exist a set of rank-1 and higher-rank solutions for the relaxed problem. To alleviate this issue, the paper [6] suggests adding a small resistance $\left(10^{-5}\right.$ per unit) to every ideal transformer with zero resistance.

Using a graph-theoretic approach combined with the SDP relaxation, we aim to study three problems:

- Since the dimension of the matrix variable $\mathbf{W}$ is prohibitive for a large-scale network, how can the computational complexity of the relaxed SCOPF be reduced?

- What is the rank of an optimal solution $\mathbf{W}_{\text {opt }}$ of the relaxed SCOPF and how does it relate to the topology of the power grid?

- If the rank of $\mathbf{W}_{\text {opt }}$ is not 1 , how can a near-global solution be recovered for the non-convex SCOPF problem?

\section{LOW-RANK SDP SOLUTIONS}

In this section, the objective is twofold. First, the computational complexity of the relaxed SCOPF will be reduced. Second, the rank of its lowest-rank solution will be studied.

\section{A. Reduction of computational complexity}

Definition 1 (Treewidth). Given a graph $\mathcal{H}=\left(\mathcal{V}_{\mathcal{H}}, \mathcal{E}_{\mathcal{H}}\right)$, a tree $\mathcal{T}$ is called a tree decomposition of $\mathcal{H}$ if it satisfies the following properties:

1) Every node of $\mathcal{T}$ corresponds to and is identified by a subset of $\mathcal{V}_{\mathcal{H}}$.

2) Every vertex of $\mathcal{H}$ is a member of at least one node of $\mathcal{T}$.

3) $\mathcal{T}_{k}$ is a connected graph for every $k \in \mathcal{V}_{\mathcal{H}}$, where $\mathcal{T}_{k}$ denotes the subgraph of $\mathcal{T}$ induced by all nodes of $\mathcal{T}$ containing the vertex $k$ of $\mathcal{H}$.

4) If $(i, j) \in \mathcal{E}_{\mathcal{H}}$, then the subgraphs $\mathcal{T}_{i}$ and $\mathcal{T}_{j}$ have at least one node in common.

Each node of $\mathcal{T}$ is a bag (collection) of vertices of $\mathcal{H}$ and hence it is referred to as bag. The width of $\mathcal{T}$ is the cardinality of its biggest bag minus one. The treewidth of $\mathcal{H}$ is the minimum width over all possible tree decompositions of $\mathcal{H}$ and is denoted by $\mathrm{tw}(\mathcal{H})$.

Note that the treewidth of a tree is equal to 1 . Figure 1 shows a graph $\mathcal{H}$ with 6 vertices named $a, b, c, d, e, f$, together with its minimal tree decomposition $\mathcal{T}$. Every node of $\mathcal{T}$ is a set containing three members of $\mathcal{V}_{\mathcal{H}}$. The width of this decomposition is therefore equal to 2 . The graph of the IEEE 14-bus system and its minimal tree decomposition are depicted in Figure 2. As shown in Table I, the treewidth of IEEE systems and various setups of Polish systems with as high as 3000 buses is at most 24 . This empirical evidence signifies that real-world power grids may have a small treewidth, which is leveraged in this work to solve the SCOPF problem.

Definition 2 (Sparsity graph). The sparsity graph of the relaxed SCOPF problem is defined as a graph with $n(c+1)$ vertices such that $(i, j)$ is an edge of the graph whenever $i \neq j, i, j \in\{1,2, \ldots, n(c+1)\}$ and $W_{i j}$ appears in either of the constraints (5a) and (5b) with a nonzero coefficient.

Consider a tree decomposition of the power network in the pre-contingency case and denote its bags (nodes) as $\mathcal{J}_{1}^{(0)}, \mathcal{J}_{2}^{(0)}, \ldots, \mathcal{J}_{p}^{(0)} \subseteq \mathcal{N}$.

\section{Theorem 1. The following statements hold:}

i) The sparsity graph of the relaxed SCOPF problem has a tree decomposition with $p(c+1)$ bags given by the set:

$$
\left\{\mathcal{J}_{m}^{(0)}+n r \mid m=1, \ldots, p \text { and } r=0, \ldots, c\right\}
$$

ii) The optimal objective value of the relaxed SCOPF problem does not change if its constraint $\mathbf{W} \succeq 0$ is replaced by

$$
\mathbf{W}\left\{\mathcal{J}_{m}^{(0)}+n r, \mathcal{J}_{m}^{(0)}+n r\right\} \succeq 0
$$

or equivalently

$$
\mathbf{W}^{(r)}\left\{\mathcal{J}_{m}^{(0)}, \mathcal{J}_{m}^{(0)}\right\} \succeq 0
$$

for every $r \in \mathcal{C}$ and $m \in\{1, \ldots, p\}$.

Proof. The proof of Part (i) follows from the fact that the sparsity graph of the relaxed SCOPF problem is composed of $c+1$ disconnected components, each corresponding to one of the contingencies $0,1,2 \ldots, c$. This is due to the fact that the constraints of the SCOPF problem can all be described in terms of only those entries of $\mathbf{W}$ that appear in one of the submatrices $\mathbf{W}^{(0)}, \ldots, \mathbf{W}^{(c)}$. Part (ii) is a direct consequence of Part (i) and the chordal theorem in [33].

Define decomposed relaxed SCOPF as a convex optimization obtained from the relaxed SCOPF by replacing $\mathbf{W} \succeq 0$ with the constraints $\mathbf{W}^{(r)}\left\{\mathcal{J}_{m}^{(0)}, \mathcal{J}_{m}^{(0)}\right\} \succeq 0$ for every $r \in \mathcal{C}$ and $m \in\{1, \ldots, p\}$. Theorem 1 reduces the computational cost of the SDP relaxation dramatically for a large-scale system with a relatively small treewidth. Note that many entries of the matrix variable $\mathbf{W}$ may not appear in the objective or constraints of the decomposed relaxed SCOPF, and those redundant entries can be eliminated. For example, the relaxed OPF for a Polish system has about 9,000,000 scalar variables, while the decomposed relaxed OPF has only about 100,000 parameters. As will be illustrated later, this enables us to solve a large-scale problem efficiently.

\section{B. Existence of low-rank solutions}

Let $\mathbf{W}_{\text {ref }} \in \mathbb{H}^{n(c+1)}$ denote an arbitrary solution of the relaxed SCOPF or decomposed relaxed SCOPF. Note that if $\mathbf{W}_{\text {ref }}$ corresponds to the decomposed problem, its redundant entries may not have been found by the numerical algorithm 
and are regarded as "missing". The following question arises: is it possible to fine-tune the entries of $\mathbf{W}_{\text {ref }}$ or design its missing entries to arrive at a different, but lower rank, solution of the (decomposed) relaxed problem? It is known that there exists a polynomial-time algorithm to fill a partially-known real-valued matrix in such a way that the rank of the resulting matrix becomes equal to the highest rank among all bags [34], [35]. We extend this result to the complex domain by proposing an iterative algorithm that transforms $\mathbf{W}_{\text {ref }}$ into a solution $\mathbf{W}_{\text {opt }}$ whose rank is upper bounded by the treewidth of the network plus one. To introduce our algorithm, consider a tree decomposition $\mathcal{T}_{\mathcal{C}}$ of the sparsity graph of the relaxed SCOPF problem with the bags specified in (6). For simplicity, we name the bags as $\mathcal{J}_{1}, \mathcal{J}_{2}, \ldots, \mathcal{J}_{p(c+1)}$.

\section{Matrix completion algorithm:}

1) Set $\mathcal{T}^{\prime}:=\mathcal{T}_{\mathcal{C}}$ and $\mathbf{W}:=\mathbf{W}_{\text {ref }}$.

2) If $\mathcal{T}^{\prime}$ has a single node, then consider $\mathbf{W}_{\text {opt }}$ as $\mathbf{W}$ and terminate; otherwise continue to the next step.

3) Choose a pair of bags $\mathcal{J}_{x}, \mathcal{J}_{y}$ of $\mathcal{T}^{\prime}$ such that $\mathcal{J}_{x}$ is a leaf of $\mathcal{T}^{\prime}$ and $\mathcal{J}_{y}$ is its unique neighbor.

4) Define

$$
\begin{aligned}
\mathbf{A} & \triangleq \mathbf{W}\left\{\mathcal{J}_{x} \cap \mathcal{J}_{y}, \mathcal{J}_{x} \cap \mathcal{J}_{y}\right\} \\
\mathbf{B}_{x} & \triangleq \mathbf{W}\left\{\mathcal{J}_{x} \backslash \mathcal{J}_{y}, \mathcal{J}_{x} \cap \mathcal{J}_{y}\right\} \\
\mathbf{B}_{y} & \triangleq \mathbf{W}\left\{\mathcal{J}_{y} \backslash \mathcal{J}_{x}, \mathcal{J}_{x} \cap \mathcal{J}_{y}\right\} \\
\mathbf{X} & \triangleq \mathbf{W}\left\{\mathcal{J}_{x} \backslash \mathcal{J}_{y}, \mathcal{J}_{x} \backslash \mathcal{J}_{y}\right\} \in \mathbb{C}^{d_{x} \times d_{x}} \\
\mathbf{Y} & \triangleq \mathbf{W}\left\{\mathcal{J}_{y} \backslash \mathcal{J}_{x}, \mathcal{J}_{y} \backslash \mathcal{J}_{x}\right\} \in \mathbb{C}^{d_{y} \times d_{y}} \\
\mathbf{S}_{x} & \triangleq \mathbf{X}-\mathbf{B}_{x} \mathbf{A}^{+} \mathbf{B}_{x}^{*}=\mathbf{Q}_{x} \boldsymbol{\Lambda}_{x} \mathbf{Q}_{x}^{*} \\
\mathbf{S}_{y} & \triangleq \mathbf{Y}-\mathbf{B}_{y} \mathbf{A}^{+} \mathbf{B}_{y}^{*}=\mathbf{Q}_{y} \boldsymbol{\Lambda}_{y} \mathbf{Q}_{y}^{*}
\end{aligned}
$$

where $\mathbf{Q}_{x} \boldsymbol{\Lambda}_{x} \mathbf{Q}_{x}^{*}$ and $\mathbf{Q}_{y} \boldsymbol{\Lambda}_{y} \mathbf{Q}_{y}^{*}$ denote the eigenvalue decompositions of $\mathbf{S}_{x}$ and $\mathbf{S}_{y}$ with the diagonals of $\boldsymbol{\Lambda}_{x}$ and $\boldsymbol{\Lambda}_{y}$ arranged in descending order. Then, update a part of $\mathbf{W}$ as follows:

$$
\begin{aligned}
\mathbf{W}\left\{\mathcal{J}_{y} \backslash \mathcal{J}_{x}, \mathcal{J}_{x} \backslash \mathcal{J}_{y}\right\}:=\mathbf{B}_{y} \mathbf{A}^{+} \mathbf{B}_{x}^{*} \\
+\mathbf{Q}_{y} \sqrt{\boldsymbol{\Lambda}_{y}} \mathbf{I}_{d_{y} \times d_{x}} \sqrt{\boldsymbol{\Lambda}_{x}} \mathbf{Q}_{x}^{*}
\end{aligned}
$$

and update $\mathbf{W}\left\{\mathcal{J}_{x} \backslash \mathcal{J}_{y}, \mathcal{J}_{y} \backslash \mathcal{J}_{x}\right\}$ accordingly to preserve the Hermitian property of $\mathbf{W}$.

5) Update $\mathcal{T}^{\prime}$ by merging $\mathcal{J}_{x}$ into $\mathcal{J}_{y}$, i.e., replace $\mathcal{J}_{y}$ with $\mathcal{J}_{x} \cup \mathcal{J}_{y}$ and then remove $\mathcal{J}_{x}$ from $\mathcal{T}^{\prime}$.

6) Go back to step 2 .

Theorem 2. Consider an arbitrary solution $\mathbf{W}_{\text {ref }}$ of the (decomposed) relaxed SCOPF problem. The output of the matrix completion algorithm, denoted as $\mathbf{W}_{\mathrm{opt}}$, is a solution of the relaxed SCOPF problem whose rank is smaller than or equal to:

$$
\max \left\{\operatorname{rank}\left\{\mathbf{W}_{\text {ref }}^{(r)}\left\{\mathcal{J}_{m}^{(0)}, \mathcal{J}_{m}^{(0)}\right\}\right\} \mid 1 \leq m \leq p, r \in \mathcal{C}\right\} .
$$

Proof. Consider one run of Step 4 of the matrix completion algorithm. Our first objective is to show that $\mathbf{W}\left\{\mathcal{J}_{x} \cup\right.$ $\left.\mathcal{J}_{y}, \mathcal{J}_{x} \cup \mathcal{J}_{y}\right\}$ is a positive semidefinite matrix whose rank is upper bounded by the maximum ranks of $\mathbf{W}\left\{\mathcal{J}_{x}, \mathcal{J}_{x}\right\}$ and
$\mathbf{W}\left\{\mathcal{J}_{y}, \mathcal{J}_{y}\right\}$. To this end, one can write:

$$
\mathbf{W}\left\{\mathcal{J}_{x} \cup \mathcal{J}_{y}, \mathcal{J}_{x} \cup \mathcal{J}_{y}\right\}=\left[\begin{array}{ccc}
\mathbf{A} & \mathbf{B}_{x}^{*} & \mathbf{B}_{y}^{*} \\
\mathbf{B}_{x} & \mathbf{X} & \mathbf{U}^{*} \\
\mathbf{B}_{y} & \mathbf{U} & \mathbf{Y}
\end{array}\right]
$$

where $\mathbf{U} \triangleq \mathbf{W}\left\{\mathcal{J}_{y} \backslash \mathcal{J}_{x}, \mathcal{J}_{x} \backslash \mathcal{J}_{y}\right\}$. Now, define

$$
\begin{aligned}
\mathbf{S}_{x y} & \triangleq\left[\begin{array}{cc}
\mathbf{X} & \mathbf{U}^{*} \\
\mathbf{U} & \mathbf{Y}
\end{array}\right]-\left[\begin{array}{c}
\mathbf{B}_{x} \\
\mathbf{B}_{y}
\end{array}\right] \mathbf{A}^{+}\left[\begin{array}{ll}
\mathbf{B}_{x}^{*} & \mathbf{B}_{y}^{*}
\end{array}\right] \\
& =\left[\begin{array}{cc}
\mathbf{Q}_{x} & \mathbf{0} \\
\mathbf{0} & \mathbf{Q}_{y}
\end{array}\right] \mathbf{M}\left[\begin{array}{cc}
\mathbf{Q}_{x}^{*} & \mathbf{0} \\
\mathbf{0} & \mathbf{Q}_{y}^{*}
\end{array}\right]
\end{aligned}
$$

where

$$
\mathbf{M} \triangleq\left[\begin{array}{ccc}
\boldsymbol{\Lambda}_{x} & \sqrt{\boldsymbol{\Lambda}_{x}} & \mathbf{I}_{d_{x} \times d_{y}} \sqrt{\boldsymbol{\Lambda}_{y}} \\
\sqrt{\boldsymbol{\Lambda}_{y}} & \mathbf{I}_{d_{y} \times d_{x}} \sqrt{\boldsymbol{\Lambda}_{x}} & \boldsymbol{\Lambda}_{y}
\end{array}\right]
$$

It is straightforward to verify that

$$
\operatorname{rank}\left\{\mathbf{S}_{x y}\right\}=\operatorname{rank}\{\mathbf{M}\}=\max \left\{\operatorname{rank}\left\{\mathbf{S}_{x}\right\}, \operatorname{rank}\left\{\mathbf{S}_{y}\right\}\right\}
$$

On the other hand, the Schur complement formula yields:

$$
\begin{aligned}
& \operatorname{rank}\left\{\mathbf{W}\left\{\mathcal{J}_{x}, \mathcal{J}_{x}\right\}\right\}=\operatorname{rank}\left\{\mathbf{A}^{+}\right\}+\operatorname{rank}\left\{\mathbf{S}_{x}\right\} \\
& \operatorname{rank}\left\{\mathbf{W}\left\{\mathcal{J}_{y}, \mathcal{J}_{y}\right\}\right\}=\operatorname{rank}\left\{\mathbf{A}^{+}\right\}+\operatorname{rank}\left\{\mathbf{S}_{y}\right\} \\
& \operatorname{rank}\left\{\mathbf{W}\left\{\mathcal{J}_{x} \cup \mathcal{J}_{y}, \mathcal{J}_{x} \cup \mathcal{J}_{y}\right\}\right\}=\operatorname{rank}\left\{\mathbf{A}^{+}\right\}+\operatorname{rank}\left\{\mathbf{S}_{x y}\right\}
\end{aligned}
$$

(see [36]). Combining the above equations leads to the conclusion that the rank of $\mathbf{W}\left\{\mathcal{J}_{x} \cup \mathcal{J}_{y}, \mathcal{J}_{x} \cup \mathcal{J}_{y}\right\}$ is upper bounded by the maximum ranks of $\mathbf{W}\left\{\mathcal{J}_{x}, \mathcal{J}_{x}\right\}$ and $\mathbf{W}\left\{\mathcal{J}_{y}, \mathcal{J}_{y}\right\}$. On the other hand, since $\mathbf{M}$ is positive semidefinite, it follows from (11) that $\mathbf{W}\left\{\mathcal{J}_{x} \cup \mathcal{J}_{y}, \mathcal{J}_{x} \cup \mathcal{J}_{y}\right\} \succeq 0$. A simple induction concludes that the output $\mathbf{W}_{\text {opt }}$ of the matrix completion algorithm is a positive semidefinite matrix whose rank is upper bounded by the expression given in the theorem. The proof is completed by noting that $\mathbf{W}_{\text {opt }}$ and $\mathbf{W}_{\text {ref }}$ share the same values on their diagonals and those off-diagonal entries corresponding to the edges of the sparsity graph of SCOPF.

Note that Theorem 2 is valid for not only relaxed SCOPF but also decomposed relaxed SCOPF. The following three results are the by-products of the above theorem.

Corollary 1. If the relaxed SCOPF problem is feasible, then it has a solution $\mathbf{W}_{\text {opt }}$ whose rank is upper bounded by the treewidth of the power network in the pre-contingency case plus 1 .

Corollary 2. For every tree network, if the relaxed SCOPF problem is feasible, then it has a solution $\mathbf{W}_{\mathrm{opt}}$ whose rank is not greater than 2.

Corollary 3. The non-convex SCOPF problem has the same globally optimal value as that of the (decomposed) relaxed SCOPF under the additional constraints

$$
\operatorname{rank}\left\{\mathbf{W}^{(r)}\left\{\mathcal{J}_{m}^{(0)}, \mathcal{J}_{m}^{(0)}\right\}\right\}=1
$$

for every $r \in \mathcal{C}$ and $m \in\{1, \ldots, p\}$.

\section{RECOVERY OF A NEAR-GLOBAL SOLUTION}

We explored the properties of the decomposed relaxed SCOPF in the preceding section. In this part, we aim to address two problems: (i) how to find a tree decomposition of the 
power network in order to be able to formulate the decomposed problem, (ii) how to recover a near-global solution of the SCOPF problem through an SDP relaxation.

\section{A. Tree decomposition algorithm}

Although the problem of finding the treewidth of an arbitrary graph is known to be NP-hard, there are many efficient algorithms in the literature that provide lower and upper bounds on treewidth [37], [38]. In what follows, we describe an effective algorithm for finding a tree decomposition that is used in all of the simulations offered in the next section. This algorithm combines the greedy degree and greedy fillin algorithms presented in [37] in order to obtain a tree decomposition for a graph with a low maximum clique order.

Consider a graph $\mathcal{H}=\left(\mathcal{V}_{\mathcal{H}}, \mathcal{E}_{\mathcal{H}}\right)$ together with an arbitrary vertex $u$ of this graph. $\delta_{\mathcal{H}}(u)$ denotes the degree of $u \in \mathcal{V}_{\mathcal{H}}$. The fill-in of $u$ is defined as the number of edges whose addition to the subgraph formed by the neighbors of $u$ makes the resulting subgraph a clique (complete subgraph). This number is denoted by $\phi_{\mathcal{H}}(u)$. The vertex $u$ is called simplicial if $\phi_{\mathcal{H}}(u)=0$ (i.e., if the neighbors of $u$ are all connected to one another).

\section{Greedy decomposition algorithm:}

1) Consider $\alpha$ as an arbitrary constant and define $\mathcal{H}^{\prime}=\mathcal{H}$. Initialize $\mathcal{T}$ as a graph with no nodes.

2) If $\mathcal{H}^{\prime}$ has a single vertex, then consider $\mathcal{T}$ as a graph with the single node $\mathcal{V}_{\mathcal{H}}$ and terminate; otherwise continue.

3) Choose a vertex $u$ in $\mathcal{H}^{\prime}$ according to the following rules:

- If $\mathcal{H}^{\prime}$ has a simplicial node, then set $u$ as that vertex.

- Otherwise, set $u$ as a (not necessarily unique) vertex of $\mathcal{H}^{\prime}$ that minimizes the function $\phi_{\mathcal{H}^{\prime}}(u)+\alpha \times$ $\delta_{\mathcal{H}^{\prime}}(u)$.

4) Define $\mathcal{U}$ as the set of all neighboring vertices of $u$ in $\mathcal{H}^{\prime}$. Add the bag $\mathcal{U} \cup\{u\}$ to $\mathcal{T}$, and then update the graph $\mathcal{H}^{\prime}$ by first connecting all vertices in $\mathcal{U}$ to each other and then removing $u$. Jump to Step 2 .

Based on [37], it is straightforward to show that a set of edges can be added to the nodes of $\mathcal{T}$ to make it a tree decomposition for $\mathcal{H}$. Since the decomposed relaxed SCOPF only needs the bags of $\mathcal{T}$, it is unnecessary to find the edges of the tree decomposition.

\section{B. Penalization method}

Consider the (decomposed) relaxed SCOPF. Since the mapping from $\mathbf{W}$ to the generating active power levels $P_{G_{k}}^{(r)}$, s is not bijective, there often exists a space of optimal matrix solutions with disparate ranks. Under such circumstance, commonly-used numerical algorithms would normally find the highest-rank SDP solution, although there may exist a hidden rank-1 solution. To address this issue in the context of OPF, we proposed in [19] to penalize the total reactive power generation $\sum_{k \in \mathcal{N}} Q_{G_{k}}$ in the objective function of the SDP relaxation. This penalty term aims to guide the numerical algorithm by speculating that the right operating point would yield a small reactive loss.
To flourish the above penalization technique, consider the case where the (decomposed) relaxed SCOPF has no rank-1 solution. Suppose that it is possible to design a convex function $g\left(\mathbf{W}^{(0)}, \ldots, \mathbf{W}^{(c)}\right)$ such that the SDP relaxation admits a rank1 solution whenever the objective of the relaxed SCOPF is replaced by this function. Then, penalizing the objective of the relaxed SCOPF with $\varepsilon \times g(\cdot)$ may lead to an approximate rank-1 SDP solution and subsequently a near-global SCOPF solution, for an appropriate choice of the penalty coefficient $\varepsilon$.

Therefore, the main challenge is to seek a penalization function $g(\cdot)$. The recent literature of compressed sensing suggests a penalty term consisting of a weighted sum of the diagonal entries of $\mathbf{W}$ [39]. However, this idea fails to work for SCOPF since all feasible solutions of the SDP relaxation have similar diagonal values due to a tight voltage control in practice. We propose a different penalty function in this paper. Consider a positive semidefinite matrix $\mathbf{X}$ with constant (fixed) diagonal entries $X_{11}, \ldots, X_{n n}$ and variable off-diagonal entries. If we maximize a weighted sum of the off-diagonal entries of $X$ with positive weights, then the $(l, m)$ entry of the optimal solution would be $X_{l m}=\sqrt{X_{l l} X_{m m}}$ for all $l, m \in\{1, \ldots, n\}$, in which case $\mathbf{X}$ becomes rank-1. Motivated by this fact, we employ the idea of elevating the off-diagonal entries of $\mathbf{W}$ to obtain a low-rank solution. For a lossless network, any decrease in the total reactive power generation increases the weighted sum of the real parts of the off-diagonal entries of $\mathbf{W}$. Likewise, the penalization of the apparent power loss over the series impedance of the lines of the network (without incorporating the shunt capacitors) plays a similar role for a lossy network. More precisely, the penalization of the loss

$$
\begin{aligned}
L_{l m}^{(r)} & \triangleq\left|S_{l m}^{(r)}+S_{m l}^{(r)}\right| \\
& =\left|V_{l}^{(r)}\left(V_{l}^{(r)}-V_{m}^{(r)}\right)^{*}+V_{m}^{(r)}\left(V_{m}^{(r)}-V_{l}^{(r)}\right)^{*}\right|\left|y_{l m}^{*}\right| \\
& =\left|W_{l l}^{(r)}+W_{m m}^{(r)}-W_{l m}^{(r)}-W_{m l}^{(r)}\right|\left|y_{l m}^{*}\right|
\end{aligned}
$$

associated with the line $(l, m)$ for contingency $r$ enforces the increase of the off-diagonal entries $W_{l m}^{(r)}$ and $W_{m l}^{(r)}$ (relative to those of $W_{l l}^{(r)}$ and $W_{m m}^{(r)}$ ). Therefore, this penalty term aims for a low-rank solution.

Penalized SDP relaxation: This optimization is obtained from the (decomposed) relaxed SCOPF problem by replacing its objective function with

$$
\sum_{\substack{k \in \mathcal{N} \\ r \in \mathcal{C}}} f_{k}^{(r)}\left(P_{G_{k}}^{(r)}\right)+\varepsilon_{b} \sum_{\substack{k \in \mathcal{N} \\ r \in \mathcal{C}}} Q_{G_{k}}^{(r)}+\varepsilon_{l} \sum_{(r, l, m) \in \mathcal{L}^{\mathrm{prob}}} L_{l m}^{(r)}
$$

for given nonnegative numbers $\varepsilon_{b}, \varepsilon_{l}$ and a set of triples $\mathcal{L}^{\text {prob }} \subseteq\left\{(r, l, m) \mid r \in \mathcal{C},(l, m) \in \mathcal{L}^{(r)}\right\}$, where $L_{l m}^{(r)}$ represents the apparent power loss over the series impedance of the line $(l, m)$ for contingency $r$.

Let $\mathbf{W}_{\text {opt }}$ and $\mathbf{W}_{\varepsilon}$ denote arbitrary solutions of the SDP and penalized SDP relaxations, respectively. Assume that $\mathbf{W}_{\text {opt }}$ does not have rank 1 , whereas $\mathbf{W}_{\varepsilon}$ has rank 1. By decomposing $\mathbf{W}_{\varepsilon}$ as $\mathbf{V}_{\varepsilon} \mathbf{V}_{\varepsilon}^{*}$, a feasible solution $\mathbf{V}_{\varepsilon}$ of the SCOPF can be obtained. In addition, the optimal value $f_{\text {opt }}$ of 
the SCOPF problem is lower bounded by the optimal value $f_{\mathrm{r} \text {-opt }}$ of the SDP relaxation and upper bounded by $f_{\varepsilon}$, where $f_{\varepsilon}$ is defined as the total generation cost associated with the operating point $\mathbf{V}_{\varepsilon}$. Define global optimality guarantee as

$$
100-\frac{f_{\varepsilon}-f_{\mathrm{r}-\mathrm{opt}}}{f_{\varepsilon}} \times 100 .
$$

This number shows the closeness of the feasible solution $\mathbf{V}_{\varepsilon}$ to the unknown globally optimal solution in terms of their costs (in percentage). For example, if the global optimality guarantee is $99 \%$, then the cost associated with the global solution of SCOPF is at most $1 \%$ better than the cost corresponding to the obtained feasible point. In summary, if the penalized SDP relaxation has a rank-1 solution, then a feasible solution of SCOPF together with a global optimality guarantee can be computed.

The success of the penalized SDP relaxation is in part related to the choice of $\mathcal{L}^{\text {prob }}$. Sometimes, a good choice is to consider this set as the collection of all lines of the system in pre- and post-contingency cases. In what follows, we propose an effective heuristic method for designing $\mathcal{L}^{\text {prob }}$. Consider a bag $\mathcal{J}_{i}$ of the tree decomposition $\mathcal{T}_{\mathcal{C}}$ together with a matrix $\mathbf{W} . \mathcal{J}_{i}$ is called a problematic bag associated with $\mathbf{W}$ if $\mathbf{W}\left\{\mathcal{J}_{i}, \mathcal{J}_{i}\right\}$ does not have rank 1 . Any line of the pre- or post-contingency network corresponding to an offdiagonal entry of $\mathbf{W}\left\{\mathcal{J}_{i}, \mathcal{J}_{i}\right\}$ is called a problematic line associated with W. It follows from Theorem 2 and Corollary 3 that the SDP relaxation is exact if there is no problematic bags/lines associated with the solution of the decomposed relaxed SCOPF

Problematic line selection algorithm: Consider the penalized SDP relaxation with $\epsilon_{l}=0$. Using a bisection method, find a value for $\epsilon_{b}$ such that the number of problematic lines associated with the penalized SDP solution is small (minimum). A candidate for $\mathcal{L}^{\text {prob }}$ is the set of resulting problematic lines.

Assume that the penalized SDP relaxation results in a rank1 solution or a low-rank matrix solution with a dominant nonzero eigenvalue. The next algorithm can be used to find an approximate feasible solution of SCOPF.

Recovery algorithm: Given a low-rank solution $\mathbf{W}_{\text {opt }}$ of the penalized SDP relaxation, we obtain an approximate solution for the SCOPF by recovering $\mathbf{V}^{(r)}$ according to the following procedure for every $r \in \mathcal{C}$ :

1) Set the voltage magnitude $V_{k}^{(r)}$ equal to the square root of the $(k, k)$ entry of $W_{\mathrm{opt}}^{(r)}$ for $k=1, \ldots, n$.

2) Find the phases of the entries of $\mathbf{V}^{(r)}$ through a convex program by minimizing

$$
\sum_{(l, m) \in \mathcal{L}^{(r)}}\left|\measuredangle\left(\mathbf{W}_{\text {opt }}\right)_{l m}^{(r)}-\measuredangle \mathbf{V}_{l}^{(r)}+\measuredangle \mathbf{V}_{m}^{(r)}\right|
$$

over the variable $\measuredangle \mathbf{V}^{(r)} \in[-\pi, \pi]^{n}$ and subject to $\measuredangle V_{1}^{(r)}=0$.

Note that the above recovery algorithm retrieves a globally optimal solution of the SCOPF problem in the case where $\operatorname{rank}\left\{\mathbf{W}_{\text {opt }}\right\}=1$. Under that circumstance, we have
$\measuredangle\left(\mathbf{W}_{\text {opt }}\right)_{l m}^{(r)}-\measuredangle \mathbf{V}_{l}^{(r)}+\measuredangle \mathbf{V}_{m}^{(r)}=0$. If the rank of $\mathbf{W}_{\text {opt }}$ is not 1 but this matrix has a dominant nonzero eigenvalue, the above recovery method aims to find a vector $\mathbf{V}$ for which the corresponding line angle differences are as closely as possible to those suggested by the matrix $\mathbf{W}_{\text {opt }}$.

\section{Simulations RESUlts}

In what follows, we offer several simulations for OPF and SCOPF problems. We have written a custom OPF Solver to perform these simulations [40], which is based on CVX and SDPT3.

For all of the cases that will be studied in this section, the penalized SDP has a rank-1 solution with $\varepsilon_{b}=0$, a roughly chosen $\varepsilon_{l}$, and

$$
\mathcal{L}^{\text {prob }}=\left\{(r, l, m) \mid r \in \mathcal{C},(l, m) \in \mathcal{L}^{(r)}\right\} .
$$

This means that the proposed method works at the first try with roughly chosen parameters, leading to a near-optimal solution. Figures 3 and 4 demonstrate for multiple systems that the near-optimal cost changes slowly by the increase of $\varepsilon_{l}$, which points to the high degree of freedom in choosing $\varepsilon_{l}$. However, a careful choice of problematic lines and the regularization parameters $\varepsilon_{b}$ and $\varepsilon_{l}$ using a bisection approach would lead to a better near-global solution. This will be elaborated in the rest of this section.

3-bus system: Consider the 3-bus system presented in [14]. The SDP relaxation may not result in a rank-1 solution for this system if a certain line is under stress (i.e. the capacity constraint of the line is binding at optimality). To address this issue, we use the penalized SDP relaxation with the objective function (15), where the parameter $\varepsilon_{b}$ is set to zero and the line under stress is chosen for penalization. The resulting optimal cost is reported in Figure 3(a) for different values of $\varepsilon_{l}$. It can be seen that there exists a relatively large interval for $\varepsilon_{l}$ that makes the penalized SDP relaxation posses a rank-1 solution with a fixed cost. This cost overlaps with the globally optimal cost of the OPF problem. Hence, our method is able to bridge the duality gap reported in [14].

IEEE and Polish systems: As our second example, we evaluate the penalization method for the OPF problem performed over benchmark systems. The results are reported in Table I. For each system, the following numbers are reported:

- Treewidth: exact treewidth or an upper bound on the treewidth (shown as " $\leq$ ") of the pre-contingency network

- Problematic bags: number of problematic bags for the SDP relaxation with $\epsilon_{b}=\epsilon_{l}=0$

- Lower bound: lower bound on the globally minimum cost of OPF, corresponding to the cost of the SDP relaxation

- Upper Bound: upper bound on the globally optimal cost of OPF, corresponding to the solution recovered from the penalized SDP problem

- Optimality guarantee: global optimality guarantee (in percentage)

- Computation time: the total computation time (in seconds) including those consumed towards tree decomposition, solving the SDP relaxation, and recovering a 


\begin{tabular}{|l|c|c|c|c|c|c|c|c|c|}
\hline $\begin{array}{c}\text { Test } \\
\text { cases }\end{array}$ & $\alpha$ & $\begin{array}{c}\text { Treewidth } \\
\text { upper bound) }\end{array}$ & $\begin{array}{c}\text { Problematic } \\
\text { bags }\end{array}$ & $\varepsilon_{b}$ & $\varepsilon_{l}$ & $\begin{array}{c}\text { Lower } \\
\text { bound }\end{array}$ & $\begin{array}{c}\text { Upper } \\
\text { bound }\end{array}$ & $\begin{array}{c}\text { Optimality } \\
\text { guarantee }\end{array}$ & $\begin{array}{c}\text { Computation } \\
\text { time (sec) }\end{array}$ \\
\hline Chow's 9 bus & 0 & 2 & 2 & 10 & 0 & 5296.68 & 5296.68 & $100 \%$ & $\leq 5$ \\
\hline IEEE 14 bus & 0 & 2 & 0 & 0 & 0 & 8081.53 & 8081.53 & $100 \%$ & $\leq 5$ \\
\hline IEEE 24 bus & 0 & 4 & 0 & 0 & 0 & 63352.20 & 63352.20 & $100 \%$ & $\leq 5$ \\
\hline IEEE 30 bus & 0 & 3 & 1 & 0.1 & 0 & 576.89 & 576.89 & $100 \%$ & $\leq 5$ \\
\hline NE 39 bus & 0 & 3 & 1 & 10 & 0 & 41862.08 & 41864.40 & $99.994 \%$ & $\leq 5$ \\
\hline IEEE 57 bus & 0 & 5 & 0 & 0 & 0 & 41737.78 & 41737.78 & $100 \%$ & $\leq 5$ \\
\hline IEEE 118 bus & 0 & 4 & 61 & 10 & 0 & 129654.61 & 129660.81 & $99.995 \%$ & $\leq 5$ \\
\hline IEEE 300 bus & 0 & 6 & 7 & 0.1 & 100 & 719711.63 & 719725.10 & $99.998 \%$ & 13.9 \\
\hline Polish 2383wp & 0 & $\leq 23$ & 651 & 3500 & 3000 & 1861510.42 & 1874322.65 & $99.316 \%$ & 529 \\
\hline Polish 2736sp & 0 & $\leq 23$ & 1 & 1500 & 0 & 1307882.29 & 1308270.20 & $99.970 \%$ & 701 \\
\hline Polish 2737sop & 0 & $\leq 23$ & 3 & 1000 & 0 & 777626.26 & 777664.02 & $99.995 \%$ & 675 \\
\hline Polish 2746wop & 0 & $\leq 23$ & 1 & 1000 & 0 & 1208273.91 & 1208453.93 & $99.985 \%$ & 801 \\
\hline Polish 2746wp & 0 & $\leq 24$ & 1 & 1000 & 0 & 1631772.83 & 1632384.87 & $99.962 \%$ & 699 \\
\hline Polish 3012wp & 1 & $\leq 24$ & 605 & 0 & 10000 & 2587740.98 & 2608918.45 & $99.188 \%$ & 814 \\
\hline Polish 3120sp & -1.5 & $\leq 24$ & 20 & 0 & 10000 & 2140765.92 & 2160800.42 & $99.073 \%$ & 910 \\
\hline
\end{tabular}

TABLE I: Performance of the penalization method for several benchmark systems.

\begin{tabular}{|l|c|c|c|c|c|c|c|}
\hline $\begin{array}{c}\text { Test } \\
\text { cases }\end{array}$ & $\begin{array}{c}\text { Local } \\
\text { minima }\end{array}$ & $\begin{array}{c}\text { Problematic } \\
\text { bags }\end{array}$ & $\varepsilon_{b}$ & $\varepsilon_{l}$ & $\begin{array}{c}\text { Lower } \\
\text { bound }\end{array}$ & $\begin{array}{c}\text { Upper } \\
\text { bound }\end{array}$ & $\begin{array}{c}\text { Optimality } \\
\text { guarantee }\end{array}$ \\
\hline WB2 & 2 & 0 & 0 & 0 & 877.78 & 877.78 & $100 \%$ \\
\hline WB3 & 2 & 0 & 0 & 0 & 417.25 & 417.25 & $100 \%$ \\
\hline WB5 & 2 & 3 & 0 & 500 & 946.53 & 946.58 & $99.995 \%$ \\
\hline WB5 Mod & 3 & 0 & 0 & 0 & 1482.22 & 1482.22 & $100 \%$ \\
\hline LMBM3 & 5 & 0 & 0 & 0 & 5694.54 & 5694.54 & $100 \%$ \\
\hline LMBM3_50 & 2 & 2 & 0 & 500 & 5789.91 & 5823.86 & $99.807 \%$ \\
\hline case22loop & 2 & 0 & 0 & 0 & 4538.80 & 4538.80 & $100 \%$ \\
\hline case30loop & 2 & 0 & 0 & 0 & 2863.06 & 2863.06 & $100 \%$ \\
\hline case30loop Mod & 3 & 0 & 0 & 0 & 2861.88 & 2861.88 & $100 \%$ \\
\hline case39 Mod4 & 3 & 4 & 1 & 0 & 557.08 & 557.15 & $99.999 \%$ \\
\hline case118 Mod1 & 3 & 36 & 10 & 0 & 129624.98 & 129625.19 & $99.999 \%$ \\
\hline case118 Mod2 & 2 & 42 & 1 & 0 & 85987.27 & 85987.59 & $100 \%$ \\
\hline case300 Mod2 & 2 & 107 & 0.5 & 50 & 474625.99 & 474643.46 & $99.996 \%$ \\
\hline
\end{tabular}

TABLE II: Performance of the penalization method on examples presented in [41].

\begin{tabular}{|l|c|c|c|c|c|c|}
\hline $\begin{array}{c}\text { Test } \\
\text { cases }\end{array}$ & $\begin{array}{c}\text { Problematic } \\
\text { bags }\end{array}$ & $\varepsilon_{b}$ & $\varepsilon_{l}$ & $\begin{array}{c}\text { Lower } \\
\text { bound }\end{array}$ & $\begin{array}{c}\text { Upper } \\
\text { bound }\end{array}$ & $\begin{array}{c}\text { Solution of } \\
\text { interior point method }\end{array}$ \\
\hline case14C & 12 & 0.1 & 10000 & 6897.02 & 7289.62 & 7238.93 \\
\hline case34tree & 1 & 0.1 & 100 & 14.49 & 24.38 & 24.38 \\
\hline
\end{tabular}

TABLE III: Performance of the penalization method on examples presented in [18].

solution (the simulations were run on a desktop computer with an Intel Core 17 quad-core $3.4 \mathrm{GHz}$ CPU and $16 \mathrm{~GB}$ RAM).

For IEEE systems, we were able to verify that the obtained tree decompositions were all minimal. Note that the permissible feasibility violation for the recovered solution was set to $10^{-6}$ for all cases reported in Table I, except for Polish 3012wp and Polish 3120sp for which the violation level was set to $1.5 \times$ $10^{-5}$. For Polish 3012wp and Polish 3120sp, we penalized the apparent power loss over all lines of each system. For IEEE 300 bus system, we penalized apparent power loss over two lines: (i) line 38 between buses 9053 and 9533, (ii) line 402 between buses 7023 and 23. These lines are problematic for the penalized SDP problem in the case $\epsilon_{b}=0.1$ and $\epsilon_{l}=0$.

Similarly, the apparent power loss penalization for Polish 2383 wp system was performed over problematic lines for the case $\epsilon_{b}=3500$ and $\epsilon_{l}=0$, leading to the following 9 lines: line 100 between buses 35 and 34, line 101 between buses 34 and 51, line 102 between buses 183 and 34, line 103 between buses 183 and 35, line 104 between buses 617 and 35, line 130 between buses 51 and 50, line 134 between buses 727 and 51, line 819 between buses 546 and 545, and line 821 between buses 727 and 545. It can be observed that the penalized SDP relaxation was able to find feasible solutions with global optimality guarantee above $99 \%$ for many benchmark examples.

New England and IEEE 300 bus systems: In order to evaluate the sensitivity of the penalization method to the choice of problematic lines and penalty parameters, we performed an experiment on New England 39 bus and IEEE 300 bus systems for the case where $\varepsilon_{b}=0$ and all lines of the network were considered in the penalty factor as "problematic". Figures 3(b) and (c) show the performance of the penalized SDP relaxation for a wide range of values for $\varepsilon_{l}$. It can be seen that it is possible to find a rank-1 solution with a high global optimality guarantee over a large interval for $\varepsilon_{l}$. However, as shown in Table I, a better optimality guarantee can be obtained by carefully tuning the penalty parameters via solving multiple SDP problems. 
Modified systems 1: We also tested the SDP relaxation method over some of the examples presented in [41]. The results are tabulated in Table II, together with the minimum number of local solutions for each system. For case WB5, line 5 (between buses 4 and 5) was chosen for apparent power loss penalization. Interestingly, the penalization of a different line in the objective function would result in the recovery of a local minimum as opposed to a global solution. For the modified IEEE 300 bus system, the apparent power loss penalization was performed over 116 problematic lines of the network. For LMBM3_50, all lines of the networks were considered as problematic.

Modified systems 2: Two OPF test cases have been recently proposed in [18], for which the semidefinite relaxation is inexact. The penalization algorithm proposed here can find a rank-1 solution for each of these cases, as reported in Table III. The test system "case34tree" is of particular interest due to its tree topology. We obtained a rank-1 solution by penalizing the only problematic line of this network.

New England system with contingency constraints: Consider the New England test system under 10 contingency scenarios, each representing the outage of one line as described in Table IV. Suppose that the objective function of the SCOPF problem only includes the power generation cost for the base case. The corrective active power production for each generator in case of contingency is set to be within $2 \mathrm{MW}$ away from the base case production level. We solve the penalized SDP relaxation by setting $\varepsilon_{b}$ to zero and minimizing the apparent power loss over all lines of the network. The result is depicted in Figure 4(a) for different values of the coefficient $\varepsilon_{l}$. It can be seen that a near-global solution for the SCOPF problem is associated with the cost 45141.70 . This SCOPF cost is $7 \%$ higher than the optimal cost of the OPF problem with no contingency.

\begin{tabular}{|c|c|c|c|}
\hline $\begin{array}{c}\text { Contingency } \\
\text { number }\end{array}$ & $\begin{array}{c}\text { Line } \\
\text { number }\end{array}$ & $\begin{array}{c}\text { Initial } \\
\text { node }\end{array}$ & $\begin{array}{c}\text { Terminal } \\
\text { node }\end{array}$ \\
\hline 1 & 1 & 1 & 2 \\
\hline 2 & 2 & 1 & 39 \\
\hline 3 & 3 & 2 & 3 \\
\hline 4 & 4 & 2 & 25 \\
\hline 5 & 6 & 3 & 4 \\
\hline 6 & 7 & 3 & 18 \\
\hline 7 & 15 & 7 & 8 \\
\hline 8 & 20 & 10 & 32 \\
\hline 9 & 40 & 25 & 26 \\
\hline 10 & 45 & 28 & 29 \\
\hline
\end{tabular}

TABLE IV: List of contingencies for New England test system.

IEEE 300 bus system with contingency constraints: Consider the 300 bus system with one contingency scenario associated with the simultaneous outage of three highly congested lines of the base OPF. These lines are listed in Table V. The corrective active power production for each generator in case of contingency is set to be within $1 \mathrm{MW}$ away from the base case production level. We intend to minimize the pre-contingency power generation cost while being secure in the post-contingency scenario. As before, we solve the penalized SDP relaxation by setting $\varepsilon_{b}$ to zero and minimizing the apparent power loss over all lines of the network. The result is depicted in Figure 4(b). It can be seen that a nearglobal solution for the SCOPF problem is associated with the cost 740493.80 , which is $3 \%$ different from that of the OPF problem.

\begin{tabular}{|c|c|c|c|}
\hline $\begin{array}{c}\text { Contingency } \\
\text { number }\end{array}$ & $\begin{array}{c}\text { Line } \\
\text { number }\end{array}$ & $\begin{array}{c}\text { Initial } \\
\text { node }\end{array}$ & $\begin{array}{c}\text { Terminal } \\
\text { node }\end{array}$ \\
\hline \multirow{3}{*}{1} & 266 & 19 & 231 \\
\cline { 2 - 4 } & 388 & 234 & 236 \\
\cline { 2 - 4 } & 400 & 7130 & 130 \\
\hline
\end{tabular}

TABLE V: List of lines outages of the contingency scenario considered for IEEE 300 bus system.

\section{CONCLUSIONS}

This paper studies the security-constrained optimal power flow (SCOPF) problem by means of a semidefinite programming (SDP) relaxation. The existence of a rank-1 solution guarantees that this convex program will find a globally optimal solution of the SCOPF problem. First, we prove that the SDP relaxation has a solution whose rank is at most equal to the treewidth of the power network plus one, which is expected to be very small for real-world systems. Second, we propose a decomposition method to reduce the computational complexity of the SDP relaxation. In the case where the SDP relaxation fails to work, we develop a graph-theoretic method to identify the problematic lines of the network that make SCOPF difficult to solve. By penalizing the loss over those lines in the SDP relaxation, we develop a rank-enforcing SDP relaxation. We test our relaxation method on several benchmark examples and demonstrate its ability in finding feasible solutions with the property that the global minima are at most $1 \%$ away from the obtained solutions.

\section{REFERENCES}

[1] J. A. Momoh, Electric Power System Applications of Optimization. New York: Markel Dekker, 2001.

[2] J. Carpentier, "Contribution to the economic dispatch problem," Bull. Soc. Francoise Elect., vol. 3, no. 8, pp. 431-447, 1962.

[3] K. Pandya and S. Joshi, "A survey of optimal power flow methods," $J$. Theoretic. Appl. Inf. Technol., vol. 4, no. 5, pp. 450-458, 2008.

[4] X. Bai, H. Wei, K. Fujisawa, and Y. Wang, "Semidefinite programming for optimal power flow problems," Int. J. Elect. Power Energy Syst., vol. 30, no. 6-7, pp. 383-392, 2008.

[5] J. Lavaei and S. H. Low, "Relationship between power loss and network topology in power systems," in Proc. IEEE 49th Conf. Decision Control, Dec. 2010, pp. 4004-4011.

[6] J. Lavaei and S. Low, "Zero duality gap in optimal power flow problem," IEEE Trans. Power Syst., vol. 27, no. 1, pp. 92-107, Feb. 2012.

[7] S. Sojoudi and J. Lavaei, "Exactness of semidefinite relaxations for nonlinear optimization problems with underlying graph structure," SIAM J. Optimiz., vol. 24, no. 4, pp. 1746-1778, Jul. 2014.

[8] J. Lavaei, B. Zhang, and D. Tse, "Geometry of power flows in tree networks," in in Proc. IEEE Power \& Energy Society General Meeting, Jul. 2012, pp. 22-27.

[9] S. Low, "Convex relaxation of optimal power flow part I: Formulations and equivalence," IEEE Trans. Power Syst., vol. 1, no. 1, pp. 15-27, Mar. 2014.

[10] S. Low, "Convex relaxation of optimal power flow part II: Exactness," IEEE Trans. Power Syst., vol. 1, no. 2, pp. 177-189, Jun. 2014.

[11] B. Zhang and D. Tse, "Geometry of injection regions of power networks," IEEE Trans. Power Syst., vol. 28, no. 2, pp. 788-797, May. 2013.

[12] S. Bose, D. F. Gayme, S. Low, and M. K. Chandy, "Optimal power flow over tree networks," in Proc. 49th Annual Allerton Conf., 2011, pp. 1342-1348. 


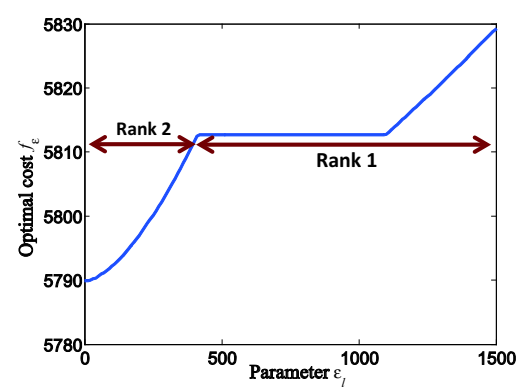

(a)

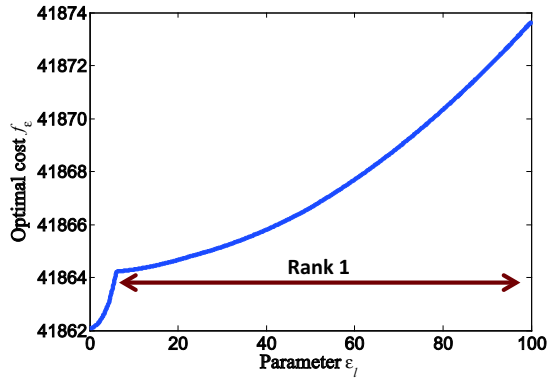

(b)

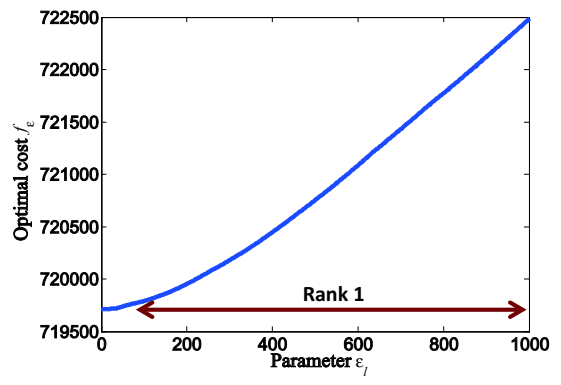

(c)

Fig. 3: (a) The 3-bus system presented in [14]; (b) New England system; (c) IEEE 300 bus system.

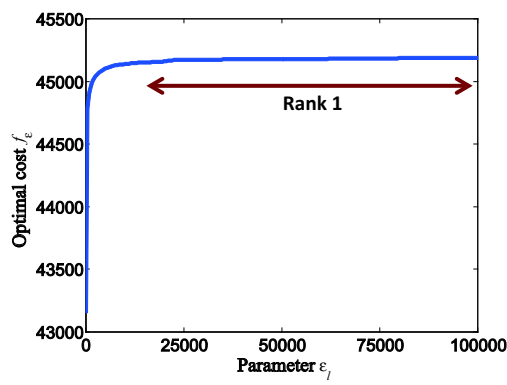

(a)

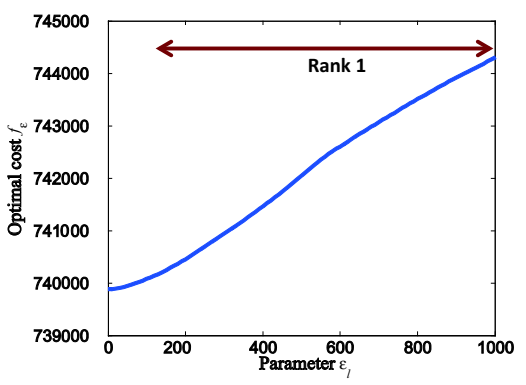

(b)

Fig. 4: (a) Contingency analysis of New England system; (b) contingency analysis of IEEE 300 bus system.

[13] S. Sojoudi and J. Lavaei, "Physics of power networks makes hard optimization problems easy to solve," IEEE Power \& Energy Society General Meeting, 2012.

[14] B. Lesieutre, D. Molzahn, A. Borden, and C. L. DeMarco, "Examining the limits of the application of semidefinite programming to power flow problems," in Proc. Allerton Conf., 2011.

[15] W. Bukhsh, A. Grothey, K. McKinnon, and P. Trodden, "Local solutions of the optimal power flow problem," IEEE Trans. Power Syst., vol. 28, no. 4, pp. 4780-4788, Nov. 2013.

[16] A. Gopalakrishnan, A. U. Raghunathan, D. Nikovski, and L. T. Biegler, "Global optimization of optimal power flow using a branch \& bound algorithm," in Proc. Allerton Conf., 2011.

[17] D. T. Phan, "Lagrangian duality and branch-and-bound algorithms for optimal power flow," Oper. Res., vol. 60, no. 2, pp. 275-285, Mar./Apr. 2012.

[18] R. Louca, P. Seiler, and E. Bitar, "Nondegeneracy and inexactness of semidefinite relaxations of optimal power flow," submitted for publication. [Online]. Available: http://arxiv.org/abs/1411.4663.

[19] R. Madani, S. Sojoudi, and J. Lavaei, "Convex relaxation for optimal power flow problem: Mesh networks," IEEE Trans. Power Syst., vol. 30, no. 1, pp. 199-211, Jan. 2015.

[20] A. Lam, B. Zhang, and D. Tse, "Distributed algorithms for optimal power flow problem," in Proc. IEEE Conf. Decision and Control, 2012.

[21] B. Zhang, A. Lam, A. Dominguez-Garcia, and D. Tse, "An optimal and distributed method for voltage regulation in power distribution systems," IEEE Trans. Power App. Syst., to be published. [Online]. Available: http://arxiv.org/abs/1204.5226.

[22] D. K. Molzahn, J. T. Holzer, B. C. Lesieutre, and C. L. DeMarco, "Implementation of a large-scale optimal power flow solver based on semidefinite programming," IEEE Trans. Power Syst., vol. 28, no. 4, pp. 3987-3998, Nov. 2013.

[23] M. S. Andersen, A. Hansson, and L. Vandenberghe, "Reducedcomplexity semidefinite relaxations of optimal power flow problems," IEEE Trans. Power Syst., vol. 29, no. 4, pp. 1855-1863, Jul 2014.

[24] R. Jabr, "Exploiting sparsity in SDP relaxations of the OPF problem," IEEE Trans. Power Syst., vol. 27, no. 2, pp. 1138-1139, May. 2012.

[25] D. K. Molzahn and I. A. Hiskens, "Sparsity-exploiting moment-based relaxations of the optimal power flow problem," IEEE Trans. Power
App. Syst., to be published. [Online]. Available: http://arxiv.org/abs/1404. 5071v2.

[26] M. Fukuda, M. Kojima, K. Murota, and K. Nakata, "Exploiting sparsity in semidefinite programming via matrix completion I: General framework," SIAM J. Optimiz., vol. 11, no. 3, pp. 647-674, 2001.

[27] K. Nakata, K. Fujisawa, M. Fukuda, M. Kojima, and K. Murota, "Exploiting sparsity in semidefinite programming via matrix completion II: Implementation and numerical results," Math. Program., vol. 95, no. 2, pp. 303-327, 2003.

[28] F. Capitanescu, M. Glavic, D. Ernst, and L. Wehenkel, "Contingency filtering techniques for preventive security-constrained optimal power flow," IEEE Trans. Power Syst., vol. 22, no. 4, pp. 1690-1697, Nov. 2007.

[29] A. J. Wood and B. F. Wollenberg, Power generation, operation, and control, 2nd ed., New York: Wiley, 1996.

[30] F. V. Fomin and D. M. Thilikos, "New upper bounds on the decomposability of planar graphs," J. Graph Theory, vol. 51, no. 1, pp. 53-81, 2006.

[31] J. Lavaei, "Zero duality gap for classical OPF problem convexifies fundamental nonlinear power problems," in Proc. Amer. Control Conf., June 2011, pp. 4566-4573.

[32] L. Gan, N. Li, U. Topcu, and S. Low, "On the exactness of convex relaxation for optimal power flow in tree networks," in Proc. 51st IEEE Conf. Decision and Control, Dec. 2012.

[33] R. Grone, C. R. Johnson, E. M. Sá, and H. Wolkowicz, "Positive definite completions of partial Hermitian matrices," Linear Algebra Appl., vol. 58, pp. 109-124, 1984.

[34] M. Laurent, "Polynomial instances of the positive semidefinite and Euclidean distance matrix completion problems," SIAM J. Matrix Aanl. A., vol. 22, no. 3, pp. 874-894, 2001.

[35] M. Laurent and A. Varvitsiotis, "A new graph parameter related to bounded rank positive semidefinite matrix completions," Math. Program., vol. 145, no. 1-2, pp. 291-325, 2014.

[36] D. Carlson, E. Haynsworth, and T. Markham, "A generalization of the Schur complement by means of the moore-penrose inverse," SIAM J. Appl. Math., vol. 26, no. 1, pp. 169-175, Jan. 1974.

[37] H. L. Bodlaender and A. M. Koster, "Treewidth computations I. upper bounds," Inform. Comput., vol. 208, no. 3, pp. 259-275, 2010. 
[38] H. L. Bodlaender and A. M. Koster, "Treewidth computations II. lower bounds," Inform. Comput., vol. 209, no. 7, pp. 1103-1119, 2011.

[39] B. Recht, M. Fazel, and P. A. Parrilo, "Guaranteed minimum rank solutions to linear matrix equations via nuclear norm minimization," SIAM Rev., vol. 52, pp. 471-501, 2010.

[40] R. Madani, M. Ashraphijuo, and J. Lavaei. (2014, Jul.) SDP Solver of Optimal Power Flow. [Online]. Available: http://www.ee.columbia.edu/ $\sim$ lavaei/Software.html

[41] W. A. Bukhsh. (2012, Feb.) Test Case Archive of Optimal Power Flow (OPF) Problems with Local Optima. [Online]. Available: http://www.maths.ed.ac.uk/OptEnergy/LocalOpt

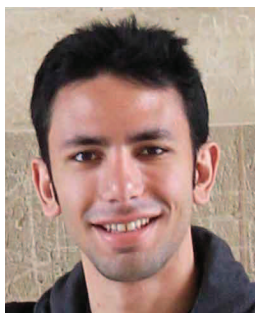

Ramtin Madani received the B.Sc. and M.Sc. degrees in electrical engineering from Sharif University of Technology, Tehran, Iran, in 2010 and 2012, respectively. $\mathrm{He}$ is currently working towards the Ph.D. degree in the Department of Electrical Engineering, Columbia University, New York, NY, USA. His current research interests include optimization over graphs, optimal power networks \& smart grids, distributed control and nonlinear optimization. $\mathrm{He}$ has worked in many areas of optimization theory, communications, and signal processing.

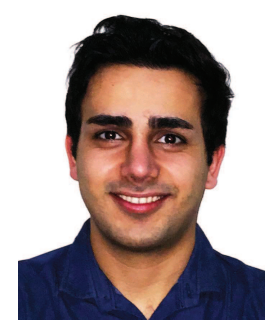

Morteza Ashraphijuo received the B.Sc. degree in electrical engineering from Sharif University of Technology, Tehran, Iran, in 2015 . He is currently working towards the Ph.D. degree in the Department of Electrical Engineering, Columbia University, New York, NY. He has won Silver Medal in the 2010 International Mathematical Olympiad. His research interests lie in the general areas of non-linear optimization with applications in power systems and control theory.

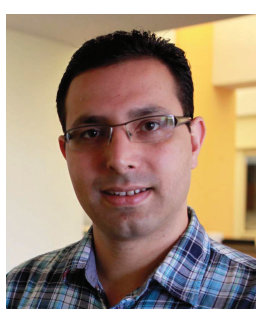

Javad Lavaei is an Assistant Professor in the Department of Electrical Engineering at Columbia University. He obtained his Ph.D. degree in Computing and Mathematical Sciences from California Institute of Technology in 2011 and held a one-year postdoc position jointly with Electrical Engineering and Precourt Institute for Energy at Stanford University. He is recipient of the Milton and Francis Clauser Doctoral Prize for the best university-wide $\mathrm{Ph} . \mathrm{D}$. thesis. His research interests include nonlinear optimization, distributed computation, power systems, and control theory. Javad Lavaei is a senior member of IEEE and has won multiple awards, including NSF CAREER Award, Office of Naval Research Young Investigator Award, Google Faculty Research Award, the Canadian Governor General's Gold Medal, Northeastern Association of Graduate Schools Master's Thesis Award, and Silver Medal in the 1999 International Mathematical Olympiad. For his computation work on energy systems, he was chosen by Resnick Sustainability Institute as one of the 5 innovators in the area of sustainability worldwide in 2014. Javad Lavaei is an associate editor for IEEE Transactions on Smart Grid and serves on the conference editorial board for both IEEE Control Systems Society and European Control Association. 